\title{
LITHIUM OBSERVATIONS IN THE SUN
}

\author{
Edith A. MÜLLER
}

The determination of the lithium abundance in the solar atmosphere is essentially based on the LiI resonance doublet at $\lambda$ 6707.761 and 6707.912 $\AA$. These two lines form a very faint absorption feature, the central depth of the stronger component being of the order of $1 \%$ of the continuum. The violet component, which is also the stronger of the two, occurs near the red wing of a faint solar line of unknown origin, and the lines appear to be blended with other faint lines including possibly the doublet of the $\mathrm{Li}^{6}$ isotope (the isotopic shift being $0 \cdot 160 \AA$ ). No other line of LiI has been detected in the Fraunhofer spectum of the undisturbed solar disk. This is nothing surprising, because practically all lithium is expected to be ionized in the photosphere on account of its low ionization potential (Xion $=5.37$ e.v.). In sunspot spectra the lower temperature reduces the degree of ionization of lithium and causes a strengthening of the LiI lines. In fact, the LiI resonance lines which appear as a very faint absorption feature on disk spectra are about 50 times stronger in spot spectra. Furthermore, the very weak feature at $\lambda 6103.6 \AA$ was identified by Dubov (1964) and by Schmahl and Schröter (1965) as due to the $2 \mathrm{~s}^{2} \mathrm{~S}-3 \mathrm{~d}{ }^{2} \mathrm{D}$ transition of LiI. Both the resonance doublet and the faint feature at $6103.6 \AA$ have been used by the above-mentioned authors to derive the lithium abundance in spots.

Since in the following paper we shall hear more about the lithium abundance in sunspots, I shall restrict myself to present the results obtained from disk spectra. In

\section{Table 1}

\section{Determinations of the solar lithium abundance}

Authors

Total $W_{\lambda}(\mathrm{m} \AA)$

Solar Model

$\log \varepsilon_{\mathrm{Li}} \mathbf{i}^{\mathrm{a}}$

Greenstein and

Richardson (1951)

Claas (1951)

Dubov (1955)

Goldberg et al. (1960)

Utrecht (1960)

Mutschlecner (1963)

Lynds (1965)

Peach (1967)

$\begin{aligned} & 3.5 \\ & 2.9 \\ & 1.74 \\ & 2.6 \\ & 2.0 \\ & 7.4 \\ &<1.6 \\ &<0.61\end{aligned}$

Milne-Eddington

Claas

$1 \cdot 26$

$1 \cdot 08$

0.93

$\begin{array}{ll}\cdots & 0.93 \\ \text { Aller-Pierce-Elste } & 0.96\end{array}$

$\begin{array}{ll}- & - \\ \text { Mutschlecner } & 1.54\end{array}$

$T=5300^{\circ}, \xi=1.4 \mathrm{~km} / \mathrm{sec}$

$<\overline{0} \cdot 38$

a On the basis of $\log \varepsilon_{\mathrm{H}}=12.00$ for the hydrogen abundance.

Perek (ed.), Highlights of Astronomy, 243-246. (C) I.A.U. 
particular, I wish to stress the following two main difficulties that are encountered when determining the lithium abundance in the undisturbed photosphere:

(1) The identification of the LiI resonance lines which is hampered by the fact that the absorption feature is faint and composite, and consequently introduces great uncertainties in the equivalent width measurements of the lithium feature.

(2) The choice of the photospheric model which by its temperature distribution in the line-forming region influences the abundance results, inasmuch as the Dopplerbroadened lithium lines are quite sensitive to the temperature.

In order to illustrate these two points the Table 1 collects the results of the equivalent width measurements, the photospheric models used, and the lithium abundance derived by different authors. The equivalent widths are given for the whole lithium feature which includes both the $\mathrm{Li}^{7}$ doublet and the corresponding isotopic $\mathrm{Li}^{6}$ doublet. The large disagreement in the equivalent widths is mainly due to identification differences, i.e. to how much of the observed absorption feature each author attributes to lithium. Apparently, as time goes on, that is with increasing spectral resolution, one finds less and less lithium in the Sun.

A high resolution spectrum of the Li I absorption feature observed at the centre of the solar disk with the Kitt Peak solar spectrograph was published by Lynds (1965). On this spectrum, two distinct small absorptions occur in the wavelength region of the $\mathrm{Li}^{7}$ and $\mathrm{Li}^{6}$ resonance doublets. However, Lynds points out that the wavelengths of the lithium lines do not coincide with the observed features and questions the presence of the lithium-absorption lines. This poor wavelength agreement was already noted by Greenstein and Richardson (1951), who interpreted it as due to pressure effects. Lynds suggests that $1.6 \mathrm{~m} \AA$ is the maximum value of the total equivalent width which may be attributed to lithium on the solar disk. Recently, Peach (1967) reinvestigated the lithium feature on high-resolution low-noise spectra. He found no direct evidence of the lithium lines in the observed absorption feature. Consequently, he gives an even smaller upper limit for the equivalent width which may be due to lithium.

Since Lynds' observation of the lithium feature is the only high-resolution spectrophotometric recording which has been published so far, we used it as a basis to derive the lithium abundance employing different photospheric models. The computations were carried out for the entire blend composed of the two $\mathrm{Li}^{7}$ and the two $\mathrm{Li}^{6}$ lines, the composite line having an equivalent width of $1.6 \mathrm{~m} \AA$. The calculations were performed with the fine analysis program of Baschek et al. (1966) and its FORTRAN translation by Peytremann. For the $\mathrm{Li}^{7} / \mathrm{Li}^{6}$ isotopic abundance ratio the following three values were used: 2,11 (corresponding approximately to the terrestrial ratio), and 20 . The four photospheric models employed and the resulting lithium abundances are given in Table 2. It is evident that different abundance results are obtained from different models. The abundance values are upper limits, inasmuch as $1.6 \mathrm{~m} \AA$ is an upper limit of the total lithium equivalent width according to Lynds. The theoretical 
Table 2

The solar lithium abundance derived for $W_{\lambda}=1.6 \mathrm{~m} \AA$ with different atmospheric models

\begin{tabular}{lc}
\multicolumn{1}{c}{ Model } & $\log \varepsilon_{\mathrm{Li}}$ \\
Holweger (1967) & $\mathbf{0 . 8 8}$ \\
Mutschlecner (1963) & $\mathbf{0 . 8 0}$ \\
U.R.M. (average column) (1964) & $\mathbf{0 . 6 2}$ \\
Heintze (1965) & 0.49
\end{tabular}

profiles best reproduced the expected lithium contribution to the observed absorption feature when setting the $\mathrm{Li}^{7} / \mathrm{Li}^{6}$ isotopic abundance ratio equal to 20 . Any large value of this ratio fits just as well. On the other hand, the small ratio of 2 produces two close lying but distinct absorption lines of almost equal central depths which is not observed. We may conclude that, if the light $\mathrm{Li}^{6}$ isotope is present, it is at least 20 times less abundant than $\mathrm{Li}^{7}$. It is interesting to note that a similar conclusion was reached by Schmahl and Schröter from sunspot spectra.

That the question of the solar abundance is by no means solved was made clear a few days ago during a session of Commission 12. L. Delbouille presented a spectrographic recording made at the Jungfraujoch of the region between $\lambda 6705$ and $6711 \AA$, in which he showed that numerous unidentified faint lines may be due to the red bands of $\mathrm{CN}$. Some of these $\mathrm{CN}$ lines fall right on the so-called lithium-absorption feature. Consequently, the lithium abundance and the $\mathrm{Li}^{7} / \mathrm{Li}^{6}$ abundance ratio must be revised, taking into account the $\mathrm{CN}$ line blending. It may be that we end up having barely any or no lithium at all present in the undisturbed photosphere.

\section{Acknowledgements}

The assistance of D. Messerschmidt (Hamburg) and E. Peytremann (Geneva) in part of the computations and the temporary use of the CERN (Geneva) and the Hamburg University (thanks to Dr. G. Traving) high-speed computers are gratefully acknowledged.

\section{References}

Baschek, B., Holweger, H., Traving, G. (1966) Abhandlg. Hamburger Sternwarte, 8, No. 1.

Claas, W.J. (1951) Rech. astr. Obs. Utrecht, 12, part 1.

Dubov, E.E. (1955) Astr. Cirk., 159, 11.

Dubov, E.E. (1964) Izv. Krym. astrofiz. Obs., 32, 26.

Goldberg, L., Müller, E. A., Aller, L.H. (1960) Astrophys. J., Suppl. Ser. 5, 1.

Greenstein, J.L., Richardson, R.S. (1951) Astrophys. J., 113, 536.

Heintze, J.R.W. (1965) Rech. astr. Obs. Utrecht, 17, (2), 1.

Holweger, H. (1967) Z. Astrophys., 65, 365.

Lynds, C. R. (1965) Astrophys. J., 142, 396.

Mutschlecner, J.P. (1963) Thesis, University of Michigan. 
Peach, J.V. (1967) Abstract of papers, 124th meeting of the Amer. Astr. Soc., p. 101.

Schmahl, G., Schröter, E.H. (1965) Z. Astrophys., 62, 143.

Utrecht (1960) 'Photometric Catalogue of Fraunhofer Lines $(\lambda \lambda 6600-8770)$ ', Rech. astr. Obs. Utrecht, 12, part 2.

U.R.M. (1964) 'Utrecht Reference Model' see: Heintze, J.R. W., Hubenet, H., and De Jager, C. (1964) Bull. astr. Inst. Netherlands, 17, 442.

\section{DISCUSSION}

L. Delbouille: Recently, high-resolution and low-noise spectra have been secured by Miss G. Roland and myself. The identifications by W. S. Benedict of many lines due to CN in the $\lambda 6708 \AA$ region of the solar spectrum seriously complicate the situation. It seems impossible to explain this region by fitting in the Lir lines only or even the Lir and the CN lines.

J.P. Mutschlecner: It should also be noted that an additional observational difficulty is the location of the local continuum. In my work, e.g., it was possible to obtain a considerable greater equivalent width due to my different interpretation of the continuum. The unidentified line at about 6707.5 also contributes to the uncertainty in the lithium lines and it would be good if it could be identified. The work of Mark Daehler (so far unpublished) might also be mentioned. In a careful re-observation and analysis he concluded that no lithium exists in the disk spectrum.

W.A. Fowler: Is there any lithium in the Sun?

L. Delbouille: As experimentalists, we try to obtain the best possible spectral tracings, but we are quite reluctant in attempting to derive an abundance of lithium for the Sun.

Mrs. Ch. Moore-Sitterly: Although there is justifiable criticism of the LiI identifications in the disk spectrum, there is no question that the resonance lines are conspicuous features in the spot spectrum. The low ionization potential of LiI makes it reasonable that LiI should be present only in the spot spectrum. Its absence from the disk spectrum may not be puzzling.

G.H. Herbig: I hardly think there can be any question that lithium exists in the solar surface. It may be marginal in the disk, but $\lambda 6707$ becomes very strong in spots $\left(W_{\lambda} \approx 50-100 \mathrm{~m} \AA\right)$ and I would not question the identification there.

H.E. Mitler: Since there is so much question as to the correct lithium abundance in the Sun, may there not be some question as to the stellar observations, especially in the low-lithium cases?

M.W. Feast: It may be as well to point out that the strength of the lithium line in the lithium lide stars is so much greater than in the Sun that we do not have in this case the kind of problems that the solar observers have.

$H$. Reeves: The lithium in sunspots may be formed right there by increased activity and may not be just strengthened over the disk spectrum by temperature effects. In this case we should expect $\mathrm{Li}^{7} / \mathrm{Li}^{6} \simeq 2$ and possibly some $\mathrm{Be}^{7}$.

Miss E.A. Müller: Due to temperature effects the LiI and the $\mathrm{CN}$ lines will be enhanced over sunspots compared to the disk spectrum, but they are expected to be strengthened differently and, hence, one might be able to separate the components. Another way of disentangling the Lit and the $\mathrm{CN}$ lines is to study the centre-to-limb variation of the $\mathrm{LiI}+\mathrm{CN}$ absorption feature and of other $\mathrm{CN}$ lines in neighbouring wavelengths pertaining to the same band. The $\mathrm{CN}$ lines are expected to have a different centre-to-limb behaviour than the Liı lines. So far, all observations discussed here refer to the centre of the solar disk. It seems to me that it would be extremely important to secure high-resolution low-noise observations of the $\mathrm{LiI}+\mathrm{CN}$ absorption feature at various positions on the solar disk and as far out to the limb as possible. If lithium is formed in sunspots, as suggested by Dr. Reeves, then one might find abundance differences in different spots depending on their activity. $\mathrm{Be}^{7}$ is probably extremely difficult to detect because the BeI and BeII lines observed in the solar spectrum occur in a big jungle of strong lines due to various atoms and molecules. 